\title{
Omni-Lie Superalgebras and Lie 2-superalgebras
}

\author{
Tao ZHANG, Zhangju LIU \\ Department of Mathematics and LMAM \\ Peking University, Beijing 100871, China
}

\begin{abstract}
We introduce the notion of omni-Lie superalgebra as a super version of the omni-Lie algebra introduced by Weinstein. This algebraic structure gives a nontrivial example of Leibniz superalgebra and Lie 2-superalgebra. We prove that there is a one-to-one correspondence between Dirac structures of the omni-Lie superalgebra and Lie superalgebra structures on subspaces of a super vector space.
\end{abstract}

\section{Introduction}

In [17], Weinstein introduced the notion of omni-Lie algebra, which can be regarded as the linearization of the Courant bracket. An omni-Lie algebra associated to a vector space $V$ is the direct sum space $\mathfrak{g l}(V) \oplus V$ together with the nondegenerate symmetric pairing $\langle\cdot, \cdot\rangle$ and the skew-symmetric bracket $\llbracket \cdot, \cdot \rrbracket$ given by

$$
\langle A+u, B+v\rangle=\frac{1}{2}(A v+B u),
$$

and

$$
\llbracket A+u, B+v \rrbracket=[A, B]+\frac{1}{2}(A v-B u) .
$$

The bracket $\llbracket \cdot, \cdot \rrbracket$ does not satisfy the Jacobi identity so that an omni-Lie algebra is not a Lie algebra. An omni-Lie algebra is actually a Lie 2-algebra since Roytenberg and Weinstein proved that every Courant algebroid gives rise to a Lie 2-algebra ([13]). Rencently, omni-Lie algebras are studied from several aspects ([4], [9], [16]) and are generalized to omni-Lie algebroids and omni-Lie 2 -algebras in $[5,6,15]$. The corresponding Dirac structures are also studied therein.

In this paper, we introduce the notion of omni-Lie superalgebra, which is the super analogue of omni-Lie algebra. We also study Dirac structures of omni-Lie superalgebra in order to characterize all possible Lie superalgebra structures on a super vector space. We prove that omni-Lie superalgebra is a Leibniz superalgebra as well as a Lie 2-superalgebra, which is a super version of Lie 2 -algebra or a 2 -term $L_{\infty}$-algebra $([2,8,10])$. 
The paper is organized as follows. In Section 2, we recall some basic facts for Lie superalgebras. In Section 3, we define omni-Lie superalgebra on $\mathcal{E}=$ $\mathfrak{g l}(V) \oplus V$ for a super vector space $V$ and study Dirac structures. In Section 4, we prove an omni-Lie superalgebra is a Lie 2-superalgebra.

\section{Lie Superalgebras and Leibniz Superalgebras}

We first recall some facts and definitions about Lie superalgebras, basic reference is Kac [7]. We work on a fixed field $\mathbb{K}$ of characteristic 0 .

A super vector space $V$ is a $\mathbb{Z}_{2}$-graded vector space with a direct sum decomposition $V=V_{\overline{0}} \oplus V_{\overline{1}}$. An element $x \in V_{\overline{0}} \cup V_{\overline{1}}$ is called homogeneous. The degree of a homogeneous element $x \in V_{\alpha}, \alpha \in \mathbb{Z}_{2}$ is defined by $|x|=\alpha$. A morphism between two super vector spaces, $V$ and $W$,is a grade-preserving linear map:

$$
f: V \longrightarrow W, \quad f\left(V_{\alpha}\right) \subseteq W_{\alpha}, \quad \forall \alpha \in \mathbb{Z}_{2} .
$$

The direct sum $V \oplus W$ is graded by

$$
(V \oplus W)_{\overline{0}}=V_{\overline{0}} \oplus W_{\overline{0}}, \quad(V \oplus W)_{\overline{1}}=V_{\overline{1}} \oplus W_{\overline{1}},
$$

and the tensor product $V \otimes W$ is graded by

$(V \otimes W)_{\overline{0}}=\left(V_{\overline{0}} \otimes W_{\overline{0}}\right) \oplus\left(V_{\overline{1}} \otimes W_{\overline{1}}\right), \quad(V \otimes W)_{\overline{1}}=\left(V_{\overline{0}} \otimes W_{\overline{1}}\right) \oplus\left(V_{\overline{1}} \otimes W_{\overline{0}}\right)$.

Definition 2.1. A Lie superalgebra is a super vector space (i.e. $\mathbb{Z}_{2}$-graded vector space) $L=L_{\overline{0}} \oplus L_{\overline{1}}$ together with a bracket $[\cdot, \cdot]: L \otimes L \rightarrow L$ satisfies,

(i) graded condition: $\left[L_{\alpha}, L_{\beta}\right] \subseteq L_{\alpha+\beta} \forall \alpha, \beta \in \mathbb{Z}_{2}$,

(ii) super skew-symmetry:

$$
[x, y]+(-1)^{|x||y|}[y, x]=0,
$$

(iii) super Jacobi identity:

$$
J_{1}:=(-1)^{|z||x|}[[x, y], z]+(-1)^{|x||y|}[[y, z], x]+(-1)^{|y||z|}[[z, x], y]=0,
$$

where $x, y, z \in L$ are homogeneous elements of degree $|x|,|y|,|z|$ respectively.

One can rewrite the super Jacobi identity in another form:

$$
J_{2}:=-(-1)^{|z||x|} J_{1}=[x,[y, z]]-[[x, y], z]-(-1)^{|x||y|}[y,[x, z]]=0,
$$

which will be convenient for our use below.

Example 2.2. Let $A=A_{\overline{0}} \oplus A_{\overline{1}}$ be an associative superalgebra with multiplication $A_{\alpha} A_{\beta} \subseteq A_{\alpha+\beta}$ for all $\alpha, \beta \in \mathbb{Z}_{2}$. Define the bracket:

$$
[x, y]:=x y-(-1)^{|x||y|} y x, \quad \forall x, y \in A .
$$

Then $(A,[\cdot, \cdot])$ is a Lie superalgebra which is denoted by $A_{L}$. 
Example 2.3. Let $V=V_{\overline{0}} \oplus V_{\overline{1}}$ be a super vector space, then we have the general linear Lie superalgebra

$$
\mathfrak{g l}(V)=\mathfrak{g l}(V)_{\overline{0}} \oplus \mathfrak{g l}(V)_{\overline{1}},
$$

such that

$$
\begin{aligned}
& \mathfrak{g l}(V)_{\overline{0}}=\operatorname{Hom}\left(V_{\overline{0}}, V_{\overline{0}}\right) \oplus \operatorname{Hom}\left(V_{\overline{1}}, V_{\overline{1}}\right), \\
& \mathfrak{g r}(V)_{\overline{1}}=\operatorname{Hom}\left(V_{\overline{0}}, V_{\overline{1}}\right) \oplus \operatorname{Hom}\left(V_{\overline{1}}, V_{\overline{0}}\right),
\end{aligned}
$$

and the bracket is given by (4). When $\operatorname{dim} V_{\overline{0}}=m$, $\operatorname{dim} V_{\overline{1}}=n, \mathfrak{g l}(V)$ is usually denoted by $\mathfrak{g l}(m \mid n)$.

A homomorphism between two Lie superalgebras $(L,[\cdot, \cdot])$ and $\left(L^{\prime},[\cdot, \cdot]^{\prime}\right)$ is linear map $\varphi: L \rightarrow L^{\prime}$ such that

$$
\varphi\left(L_{\alpha}\right) \subseteq L_{\alpha}^{\prime}, \quad \varphi([x, y])=[\varphi(x), \varphi(y)]^{\prime}, \quad \forall x, y \in L, \forall \alpha \in \mathbb{Z}_{2} .
$$

A super vector space $V=V_{\overline{0}} \oplus V_{\overline{1}}$ is called a module of a Lie superalgebra $L$ or, equivalently, say that $L$ acts on $V$ if there is a homomorphism $\rho: L \rightarrow \mathfrak{g l}(V)$, i.e.,

$$
\rho([x, y]) v=\rho(x) \rho(y) v-(-1)^{|x||y|} \rho(y) \rho(x) v .
$$

For simplicity, one often writes $x v=\rho(x) v$ to denote such an action. A new Lie superalgebra can constructed as follows.

Proposition 2.4. [14] Let $L$ be a Lie superalgebra with an action on $V$. Define a bracket on $L \oplus V$ by

$$
[x+u, y+v]:=[x, y]_{L}+x v-(-1)^{|u||y|} y u .
$$

Then $(L \oplus V,[\cdot, \cdot])$ becomes a Lie superalgebra, denoted by $L \ltimes V$ and called semidirect product of $L$ and $V$.

In [12], Loday introduced a new algebraic structure, which is usually called Leibniz algebra. Its super version is as follows.

Definition 2.5. [1] A Leibniz superalgebra is a super vector space $L=L_{\overline{0}} \oplus L_{\overline{1}}$ together with a morphism $\circ: L \otimes L \rightarrow L$ satisfying $L_{\alpha} \circ L_{\beta} \subseteq L_{\alpha+\beta}$ for all $\alpha, \beta \in \mathbb{Z}_{2}$, and the super Leibniz rule:

$$
x \circ(y \circ z)=(x \circ y) \circ z+(-1)^{|x||y|} y \circ(x \circ z),
$$

for all homogeneous elements $x, y, z \in L$.

By definition, it is easy to see that a Leibniz superalgebra is just a Lie superalgebra if the operation " 0 " is super skew-symmetric. In this case, the super Leibniz rule above is actually the super Jacobi identity for $J_{2}$ given by (3). 


\section{Omni-Lie Superlgebras and Dirac Stuctures}

Let $V$ be a super vector space, recall that the space $\mathfrak{g l}(V) \oplus V$ has a $\mathbb{Z}_{2}$-grading

$$
\mathcal{E}:=\mathfrak{g l}(V) \oplus V=\left(\mathfrak{g l}(V)_{\overline{0}} \oplus V_{\overline{0}}\right) \oplus\left(\mathfrak{g l}(V)_{\overline{1}} \oplus V_{\overline{1}}\right) .
$$

Like in [9], we define an operation $\circ$ on $\mathfrak{g l}(V) \oplus V$ as follows:

$$
(A+x) \circ(B+y)=[A, B]+A y
$$

Then we have

Proposition 3.1. ( $\mathcal{E}, \circ)$ is a Leibniz superalgebra.

Proof. To check the super Leibniz rule (7) holds on $\mathcal{E}$ under the operation $\circ$, let $e_{1}=A+x, e_{2}=B+y, e_{3}=C+z$ be homogenous elements of degree $|A|=|x|$, $|B|=|y|$ and $|C|=|z|$. By definition, we have

$$
\begin{aligned}
& \left\{e_{1} \circ e_{2}\right\} \circ e_{3}-e_{1} \circ\left\{e_{2} \circ e_{3}\right\}-(-1)^{|x||y|} e_{2} \circ\left\{e_{1} \circ e_{3}\right\} \\
= & ([A, B]+A y) \circ(C+z)-(A+x) \circ([B, C]+B z) \\
& -(-1)^{|x||y|}(B+y) \circ([A, C]+A z) \\
= & {[[A, B], C]-[A,[B, C]]-(-1)^{|x||y|}[B,[A, C]] } \\
& +[A, B] z-A B z-(-1)^{|x||y|} B A z \\
= & 0
\end{aligned}
$$

where the equality holds because $\mathfrak{g l}(V)$ is a Lie superalgebra acting on $V$.

Note that the above operation is not super skew-symmetric, we can define a super skew-symmetric bracket on $\mathcal{E}=\mathfrak{g l}(V) \oplus V$ as its skew symmetrization:

$$
\begin{aligned}
\llbracket A+x, B+y \rrbracket & \triangleq \frac{1}{2}((A+x) \circ(B+y)-(B+y) \circ(A+x)) \\
& =[A, B]+\frac{1}{2}\left(A y-(-1)^{|x||y|} B x\right)
\end{aligned}
$$

and define a $V$-valued inner product, i.e., a non-degenerated super symmetric bilinear form:

$$
\langle A+x, B+y\rangle \triangleq \frac{1}{2}\left(A y+(-1)^{|x||y|} B x\right) .
$$

We call the triple $(\mathcal{E}, \llbracket \cdot, \cdot \rrbracket,\langle\cdot, \cdot\rangle)$ an omni-Lie superalgebra. Without the factor $1 / 2$ in bracket $\llbracket \cdot, \cdot \rrbracket$, this would be the semidirect product Lie superalgebra for the action of $\mathfrak{g l}(V)$ on $V$ described in Proposition 2.4. With the factor $1 / 2$, the bracket does not satisfy the super Jacobi identity, which leads to the concept of Lie 2-superalgebra defined in the next section. Next we compute the Jacobiator for this bracket.

Proposition 3.2. For $e_{1}=A+x, e_{2}=B+y, e_{3}=C+z \in \mathcal{E}$, define

$$
\begin{aligned}
T\left(e_{1}, e_{2}, e_{3}\right):= & \frac{1}{3}\left\{(-1)^{|z||x|}\left\langle\llbracket e_{1}, e_{2} \rrbracket, e_{3}\right\rangle+(-1)^{|x||y|}\left\langle\llbracket e_{2}, e_{3} \rrbracket, e_{1}\right\rangle\right. \\
& \left.+(-1)^{|y||z|}\left\langle\llbracket e_{3}, e_{1} \rrbracket, e_{2}\right\rangle\right\} .
\end{aligned}
$$


Let $J_{1}$ denote the Jacobiator given in (2) for the bracket $\llbracket \cdot, \cdot \rrbracket$ on $\mathcal{E}$, then we have

$$
J_{1}\left(e_{1}, e_{2}, e_{3}\right)=T\left(e_{1}, e_{2}, e_{3}\right) .
$$

Proof. We compute both the sides as follows:

$$
\begin{aligned}
& J_{1}\left(e_{1}, e_{2}, e_{3}\right) \\
= & (-1)^{|z||x|} \llbracket \llbracket A+x, B+y \rrbracket, C+z \rrbracket+\text { c.p. } \\
= & \llbracket(-1)^{|z| x \mid}[A, B]+\frac{1}{2}(-1)^{|z||x|}\left(A y-(-1)^{|x||y|} B x\right), C+z \rrbracket+\text { c.p. } \\
= & (-1)^{|z||x|}[[A, B], C]+\text { c.p. } \\
& +\frac{1}{2}\left((-1)^{|z||x|}[A, B] z-\frac{1}{2}(-1)^{|z||x|}(-1)^{(|x|+|y|)|z|} C\left(A y-(-1)^{|x||y|} B x\right)\right) \\
& +\frac{1}{2}\left((-1)^{|x||y|}[B, C] x-\frac{1}{2}(-1)^{|x||y|}(-1)^{(|y|+|z|)|x|} A\left(B z-(-1)^{|y||z|} C y\right)\right) \\
& +\frac{1}{2}\left((-1)^{|y||z|}[C, A] y-\frac{1}{2}(-1)^{|z||y|}(-1)^{|| z|+| x \mid)|y|} B\left(C x-(-1)^{|z||x|} A z\right)\right) \\
= & \frac{1}{4}(-1)^{|z||x|} A B z-\frac{1}{4}(-1)^{|z||x|}(-1)^{|x||y|} B A z+\frac{1}{4}(-1)^{|y||z|} C A y \\
& -\frac{1}{4}(-1)^{|y||z|}(-1)^{|x| y \mid} C B x+\frac{1}{4}(-1)^{|x||y|} B C x-\frac{1}{4}(-1)^{|z||x|}(-1)^{|y||z|} A C y, \\
& T\left(e_{1}, e_{2}, e_{3}\right) \\
= & \frac{1}{3}(-1)^{|z||x|}\langle\llbracket A+x, B+y \rrbracket, C+z\rangle+\mathrm{c} \cdot \mathrm{p} . \\
= & \frac{1}{3}(-1)^{|z||x|}\left\langle[A, B]+\frac{1}{2}\left(A y-(-1)^{|x||y|} B x\right), C+z\right\rangle+\mathrm{c.p.} . \\
= & \frac{1}{6}(-1)^{|z||x|}\left([A, B] z+\frac{1}{2}(-1)^{(|x|+|y|)|z|} C\left(A y-(-1)^{|x||y|} B x\right)\right)+\mathrm{c.p.} . \\
= & \frac{1}{6}(-1)^{|z||x|} A B z-\frac{1}{6}(-1)^{|z||x|}(-1)^{|x||y|} B A z+\frac{1}{12}(-1)^{|y||z|} C A y \\
& -\frac{1}{12}(-1)^{|y||z|}(-1)^{|x||y|} C B x+\frac{1}{6}(-1)^{|x||y|} B C x-\frac{1}{6}(-1)^{|x||y|}(-1)^{|y||z|} C B x \\
& +\frac{1}{12}(-1)^{|z||x|} A B z-\frac{1}{12}(-1)^{|z||x|}(-1)^{|y||z|} A C y+\frac{1}{6}(-1)^{|y||z|} C A y \\
& -\frac{1}{6}(-1)^{|y||z|}(-1)^{|z||x|} A C y+\frac{1}{12}(-1)^{|x||y|} B C x-\frac{1}{12}(-1)^{|x||y|}(-1)^{|z||x|} B A z \\
= & \frac{1}{4}(-1)^{|z||x|} A B z-\frac{1}{4}(-1)^{|z||x|}(-1)^{|x||y|} B A z+\frac{1}{4}(-1)^{|y||z|} C A y \\
& -\frac{1}{4}(-1)^{|y||z|}(-1)^{|x||y|} C B x+\frac{1}{4}(-1)^{|x||y|} B C x-\frac{1}{4}(-1)^{|z||x|}(-1)^{|y||z|} A C y .
\end{aligned}
$$

Thus, the two sides are equal.

The bracket $\llbracket \cdot, \cdot \rrbracket$ does not satisfy the super Jacobi identity so that an omniLie superalgebra is not a Lie superalgebra. However, all possible Lie superalgebra structures on $V$ can be characterized by means of the omni-Lie superalgebra.

For a bilinear operation $\omega$ on $V$ such that $\omega: V_{\alpha} \times V_{\beta} \rightarrow V_{\alpha+\beta}$, we define the adjoint operator

$$
\operatorname{ad}_{\omega}: V_{\alpha} \rightarrow \mathfrak{g l}(V)_{\alpha}, \quad \operatorname{ad}_{\omega}(x)(y)=\omega(x, y) \in V_{\alpha+\beta}
$$

where $x \in V_{\alpha}, y \in V_{\beta}$. Then the graph of the adjoint operator:

$$
\mathcal{F}_{\omega}=\left\{\operatorname{ad}_{\omega} x+x ; \forall x \in V\right\} \subset \mathcal{E}=\mathfrak{g l}(V) \oplus V
$$

is a super subspace of $\mathcal{E}$. Denote $\mathcal{F}_{\omega}^{\perp}$ the orthogonal complement of $\mathcal{F}_{\omega}$ in $\mathcal{E}$ with respect to the super symmetric bilinear form $\langle\cdot, \cdot\rangle$ on $\mathcal{E}$ given in (10). 
Proposition 3.3. With the above notations, $(V, \omega)$ is a Lie superalgebra if and only if its graph $\mathcal{F}_{\omega}$ is maximal isotropic, i.e. $\mathcal{F}_{\omega}=\mathcal{F}_{\omega}^{\perp}$, and is closed with respect to the bracket $\llbracket \cdot, \cdot \rrbracket$.

Proof. First we see that

$$
\begin{aligned}
\left\langle\operatorname{ad}_{\omega}(x)+x, \operatorname{ad}_{\omega}(y)+y\right\rangle & =\frac{1}{2}\left(\operatorname{ad}_{\omega}(x) y+(-1)^{|x||y|} \operatorname{ad}_{\omega}(y) x\right) \\
& =\frac{1}{2}\left(\omega(x, y)+(-1)^{|x||y|} \omega(y, x)\right) .
\end{aligned}
$$

This means that $\omega$ is super skew-symmetric if and only if its graph is isotropic, i.e. $\mathcal{F}_{\omega} \subseteq \mathcal{F}_{\omega}^{\perp}$. Moreover, by dimension analysis, we have $\mathcal{F}_{\omega}$ is maximal isotropic.

Next let $[x, y]:=\omega(x, y)$, we shall check that the super Jacobi identity on $V$ is satisfied if and only if $\mathcal{F}_{\omega}$ is closed under bracket (9) on $\mathcal{E}$. In fact,

$$
\begin{aligned}
\llbracket \operatorname{ad}_{\omega}(x)+x, \operatorname{ad}_{\omega}(x)+y \rrbracket & =\left[\operatorname{ad}_{\omega}(x), \operatorname{ad}_{\omega}(y)\right]+\frac{1}{2}\left(\operatorname{ad}_{\omega}(x) y-(-1)^{|x||y|} \operatorname{ad}_{\omega}(y) x\right) \\
& =\left[\operatorname{ad}_{\omega}(x), \operatorname{ad}_{\omega}(y)\right]+\frac{1}{2}\left(\omega(x, y)-(-1)^{|x||y|} \omega(y, x)\right) \\
& =\left[\operatorname{ad}_{\omega}(x), \operatorname{ad}_{\omega}(y)\right]+\omega(x, y) .
\end{aligned}
$$

Thus this bracket is closed if and only if

$$
\left[\operatorname{ad}_{\omega}(x), \operatorname{ad}_{\omega}(y)\right]=\operatorname{ad}_{\omega}(\omega(x, y)) .
$$

In this case, for $\forall z \in V$, we have

$$
\begin{aligned}
& {\left[\operatorname{ad}_{\omega}(x), \operatorname{ad}_{\omega}(y)\right](z)-\operatorname{ad}_{\omega}(\omega(x, y))(z) } \\
= & \operatorname{ad}_{\omega}(x) \operatorname{ad}_{\omega}(y)(z)-(-1)^{|x||y|} \operatorname{ad}_{\omega}(y) \operatorname{ad}_{\omega}(x)(z)-\operatorname{ad}_{\omega}(\omega(x, y))(z) \\
= & \operatorname{ad}_{\omega}(x) \omega(y, z)-(-1)^{|x||y|} \operatorname{ad}_{\omega}(y) \omega(x, z)-\omega(\omega(x, y), z) \\
= & \omega(x, \omega(y, z))-(-1)^{|x||y|} \omega(y, \omega(x, z))-\omega(\omega(x, y), z) \\
= & {[x,[y, z]]-(-1)^{|x||y|}[y,[x, z]]-[[x, y], z] } \\
= & 0 .
\end{aligned}
$$

This is exactly the super Jacobi identity on $V$. Therefore, the conclusion follows from Definition 2.1.

In [3], quadratic Lie superalgebras are studied for a given inner product $B$ on $V$. In this case, one has the orthogonal Lie superalgebra $\mathfrak{o}(V) \subset \mathfrak{g l}(V)$ and it is easy to see that $(V, \omega, B)$ is a quadratic Lie superalgebra if and only if $\omega$ satisfies the two conditions in Proposition 3.3 above as well as $\operatorname{ad}_{\omega} x \in \mathfrak{o}(V), \forall x \in V$.

Definition 3.4. A Dirac structure $L$ of the omni-Lie superalgebra $(\mathfrak{g l}(V) \oplus$ $V, \llbracket \cdot, \cdot \rrbracket,\langle\cdot, \cdot\rangle)$ is a maximal isotropic subspace $\left(L=L^{\perp}\right)$ and closed under the bracket $\llbracket \cdot, \cdot \rrbracket$.

Remark 3.5. According to Proposition 3.2, for a Dirac structure L, we have

$$
J_{1}\left(e_{1}, e_{2}, e_{3}\right)=T\left(e_{1}, e_{2}, e_{3}\right)=0, \quad \forall e_{i} \in L .
$$


Thus a Dirac structure is a Lie superalgebra, though omni-Lie superalgebra is not for itself. In fact, a Dirac structure is also a Leibniz subalgebra under the operation $\circ$.

By Proposition 3.3, $(V, \omega)$ is a Lie superalgebras if and only if $\mathcal{F}_{\omega}$ is a Dirac structure of the omni-Lie superalgebra $\mathfrak{g l}(V) \oplus V$. In order to give a general characterization for all Dirac structures of $\mathcal{E}$, we adapt the theory of characteristic pairs developed in [11] (see also [15]).

For a maximal isotropic subspace $L \subset \mathfrak{g l}(V) \oplus V$, set the subspace $D=$ $L \cap \mathfrak{g l}(V)$. Define $D^{0} \subset V$ to be the null space of $D$ :

$$
D^{0}=\{x \in V \mid X(x)=0, \forall X \in D\} .
$$

It is easy to see that $D=\left(D^{0}\right)^{0}$.

Lemma 3.6. With notations above, a subspace $L$ is maximal isotropic if and only if $L$ is of the form

$$
L=D \oplus \mathcal{F}_{\left.\pi\right|_{D^{0}}}=\left\{X+\pi(x)+x \mid X \in D, x \in D^{0}\right\},
$$

where $\pi: V \rightarrow \mathfrak{g l}(V)$ is a super skew-symmetric map.

Proof. In the following, we also denote $\pi(x, y)=\pi(x)(y) \in V$ for convenience. First suppose that $L$ is given by (11), then

$$
\begin{aligned}
& \langle X+\pi(x)+x, Y+\pi(y)+y\rangle \\
= & \frac{1}{2}\left\{X(y)+\pi(x, y)+(-1)^{|x||y|} Y(x)+(-1)^{|x||y|} \pi(y, x)\right\} \\
= & \frac{1}{2}\left\{\pi(x, y)+(-1)^{|x||y|} \pi(y, x)\right\} \\
= & 0, \quad \forall X+\pi(x)+x, Y+\pi(y)+y \in L,
\end{aligned}
$$

since $\pi: V \rightarrow \mathfrak{g l}(V)$ is super skew-symmetric so that $L$ is isotropic. Next we prove that $L$ is maximal isotropic. For $\forall Z+z \in L^{\perp}$,

$$
\langle X, Z+z\rangle=X(z)=0, \quad \forall X \in D \Rightarrow z \in D^{0} .
$$

Moreover, $\forall x \in D^{0}$, the equality belew

$$
\begin{aligned}
\langle X+\pi(x)+x, C+z\rangle & =X(z)+\pi(x)(z)+(-1)^{|x||z|} C x \\
& =(-1)^{|x||z|}(C-\pi(z))(x)=0,
\end{aligned}
$$

implies that $C-\pi(z) \triangleq Z \in D$. Thus

$$
C+z=Z+\pi(z)+z \in L=D \oplus \mathcal{F}_{\left.\pi\right|_{D^{0}}} \Rightarrow L=L^{\perp} .
$$

The converse part is straightforward so we omit the details.

The proof of the following Lemma is skipped since it is straightforward and similar to that in [15]. 
Lemma 3.7. Let $(D, \pi)$ be given above. Then $L$ is a Dirac structure if and only if the following conditions are satisfied:

(1) $D$ is a subalgebra of $\mathfrak{g l}(V)$;

(2) $\pi(\pi(x, y))-[\pi(x), \pi(y)] \in D, \quad \forall x, y \in D^{0}$;

(3) $\pi(x, y) \in D^{0}, \quad \forall x, y \in D^{0}$.

Such a pair $(D, \pi)$ is called a characteristic pair of a Dirac structure $L$. By means of the two lemmas above, we can mention the main result in this section.

Theorem 3.8. There is a one-to-one correspondence between Dirac structures of the omni-Lie superalgebra $(\mathfrak{g l}(V) \oplus V,\langle\cdot, \cdot\rangle, \llbracket \cdot, \cdot \rrbracket)$ and Lie superalgebra structures on subspaces of $V$.

Proof. For any Dirac structure $L=D \oplus \mathcal{F}_{\left.\pi\right|_{D^{0}}}$, a Lie superalgebra structure on $D^{0}$ is as follows:

$$
[x, y]_{D^{0}} \triangleq \pi(x, y) \in D^{0}, \quad \forall x, y \in D^{0} .
$$

It easy to see that this is a super skew-symmetric map. For super Jacobi indentity, we have for all $x, y, z \in D^{0}$,

$$
\begin{aligned}
{\left[[x, y]_{D^{0}}, z\right]_{D^{0}} } & =\pi\left([x, y]_{D^{0}}\right)(z)=\pi((\pi(x)(y))(z)=[\pi(x), \pi(y)](z) \\
& =\pi(x)(\pi(y)(z))-(-1)^{|x||y|} \pi(y)(\pi(x)(z)) \\
& =\left[x,[y, z]_{D^{0}}\right]_{D^{0}}-(-1)^{|x||y|}\left[y,[x, z]_{D^{0}}\right]_{D^{0}} .
\end{aligned}
$$

Thus we get a Lie superalgebra $\left(D^{0},[\cdot, \cdot]_{D^{0}}\right)$.

Conversely, for any Lie superalgebra $\left(W,[\cdot, \cdot]_{W}\right)$ on a subspace $W$ of $V$. Define $D$ by

$$
D=W^{0} \triangleq\{X \in \mathfrak{g l}(V) \mid X(x)=0, \quad \forall x \in W\} .
$$

Then $D^{0}=\left(W^{0}\right)^{0}=W$. Since Lie superalgebra structure $[\cdot, \cdot]_{W}$ gives a super skew symmetric morphism:

$$
\operatorname{ad}: W \rightarrow \mathfrak{g l}(W), \quad \operatorname{ad}_{x}(y)=[x, y]_{W},
$$

we take a super skew symmetric morphism $\pi: V \rightarrow \mathfrak{g l}(V)$, as an extension of ad from $W=D^{0}$ to $V$. Thus we get a maximal isotropic subspace $L=D \oplus \mathcal{F}_{\left.\pi\right|_{W}}$ from the pair $(D, \pi)$ as in Lemma 3.6.

We shall prove that $L$ is a Dirac structure. Firstly, $\forall X, Y \in D$ and $x \in W$, we have

$$
[X, Y](x)=X Y(x)-(-1)^{|X||Y|} Y X(x)=0,
$$

which implies that $D$ is a subalgebra of $\mathfrak{g l}(V)$. 
Next step is to prove that $L$ is closed under the bracket $\llbracket \cdot, \cdot \rrbracket$. Remember that $\left.\pi\right|_{W}=$ ad and $[\cdot, \cdot]_{W}$ satisfies the super Jacobi identity, we obtain

$$
\left[\operatorname{ad}_{x}, \operatorname{ad}_{y}\right]=\operatorname{ad}_{[x, y]_{W}}=\operatorname{ad}_{a d_{x} y}, \quad \forall x, y \in W .
$$

For any $X \in D$ and $x, y \in W$, we have

$$
\left[X, \operatorname{ad}_{x}\right](y)=X\left([x, y]_{W}\right)-(-1)^{|X||x|}[x, X(y)]=0,
$$

thus $\left[X, \operatorname{ad}_{x}\right] \in D$. On the other hand, we have

$$
\begin{aligned}
& \llbracket X+\operatorname{ad}_{x}+x, Y+\operatorname{ad}_{y}+y \rrbracket \\
= & {[X, Y]+\left[X, \operatorname{ad}_{y}\right]+\left[\operatorname{ad}_{x}, Y\right]+\left[\operatorname{ad}_{x}, \operatorname{ad}_{y}\right]+\frac{1}{2}\left(\operatorname{ad}_{x}(y)-(-1)^{|x||y|} \operatorname{ad}_{y}(x)\right) } \\
= & {[X, Y]+\left[X, \operatorname{ad}_{y}\right]+\left[\operatorname{ad}_{x}, Y\right]+\operatorname{ad}_{[x, y]_{W}}+[x, y]_{W} } \\
\in & D \oplus \mathcal{F}_{\pi_{\left.\right|_{W}}},
\end{aligned}
$$

Thus, we conclude that $L$ is a Dirac structure. Finally, it is easy to see that the Dirac structure $L$ is independent of the choice of extension $\pi$. This completes the proof.

\section{Lie 2-superalgebras}

The concept of Lie $n$-superalgebras is introduced in [8]. In particular, the axiom of a Lie 2-superalgebra can be expressed explicitly as follows:

Definition 4.1. A Lie 2-superalgebra $\mathcal{V}=\left(\mathcal{V}^{1} \stackrel{d}{\longrightarrow} \mathcal{V}^{0}, l_{2}, l_{3}\right)$ consists of the following data:

- two super vector spaces $\mathcal{V}^{0}$ and $\mathcal{V}^{1}$ together with a morphism $d: \mathcal{V}^{1} \rightarrow \mathcal{V}^{0}$;

- a morphism $l_{2}=[\cdot, \cdot]: \mathcal{V}^{i} \otimes \mathcal{V}^{j} \rightarrow \mathcal{V}^{i+j}$;

- a morphism $l_{3}: \mathcal{V}^{0} \otimes \mathcal{V}^{0} \otimes \mathcal{V}^{0} \rightarrow \mathcal{V}^{1}$;

such that, $\forall x, y, z, w \in \mathcal{V}^{0} ; \forall h, k \in \mathcal{V}^{1}$,

(a) $[x, y]+(-1)^{|x||y|}[y, x]=0$;

(b) $[x, h]+(-1)^{|x||h|}[h, x]=0$;

(c) $[h, k]=0$;

(d) $l_{3}(x, y, z)$ is totally super skew-symmetric;

(e) $d([x, h])=[x, d h]$;

(f) $[d h, k]=[h, d k]$;

(g) $d\left(l_{3}(x, y, z)\right)=-[[x, y], z]+[x,[y, z]]+(-1)^{|y||z|}[[x, z], y]$; 
(h) $l_{3}(x, y, d h)=-[[x, y], h]+[x,[y, h]]+(-1)^{|y||h|}[[x, z], h]$;

(i) $\delta l_{3}(x, y, z, w):=\left[x, l_{3}(y, z, w)\right]-(-1)^{|x||y|}\left[y, l_{3}(x, z, w)\right]$

$$
\begin{aligned}
& +(-1)^{(|x|+|y|)|z|}\left[z, l_{3}(x, y, w)\right]-\left[l_{3}(x, y, z), w\right]-l_{3}([x, y], z, w) \\
& +(-1)^{|y||z|} l_{3}([x, z], y, w)-(-1)^{(|y|+|z|)|w|} l_{3}([x, w], y, z) \\
& -l_{3}(x,[y, z], w)+(-1)^{|z||w|} l_{3}(x,[y, w], z)-l_{3}(x, y,[z, w])=0 .
\end{aligned}
$$

This is the super analogue of a 2 -term $L_{\infty}$-algebra which is equivalent to a Lie 2-algebra (see [2] for more details). Here we use the terminology Lie 2 -superalgebra instead of 2-term $L_{\infty}$-superalgebra.

Now, for a super vector space $V$, let

$$
\mathcal{V}^{0}=\mathfrak{g l}(V) \oplus V, \quad \mathcal{V}^{1}=V, \quad d=i: V \hookrightarrow \mathfrak{g l}(V) \oplus V,
$$

where $i$ is the inclusion map and define operations:

$$
l_{2}=\llbracket \cdot, \cdot \rrbracket, \quad l_{3}=-(-1)^{|z||x|} T .
$$

Theorem 4.2. With notations above, the omni-Lie superalgebra $(\mathcal{E}, \llbracket \cdot, \cdot \rrbracket,\langle\cdot, \cdot\rangle)$ defines a Lie 2-superalgebra $\left(V \stackrel{d}{\hookrightarrow} \mathfrak{g l}(V) \oplus V, l_{2}, l_{3}\right)$.

Proof. For Condition $(a)$, by the grading in $\mathfrak{g l}(V) \oplus V$ we have $\operatorname{deg}(A+x)=$ $\operatorname{deg}(A)=\operatorname{deg}(x)$, then

$$
\begin{aligned}
& \llbracket A+x, B+y \rrbracket+(-1)^{|x||y|} \llbracket B+y, A+x \rrbracket \\
= & {[A, B]+\frac{1}{2}\left(A y-(-1)^{|x||y|} B x\right)+(-1)^{|A||B|}[B, A] } \\
& +(-1)^{|x||y|} \frac{1}{2}\left(B x-(-1)^{|y||x|} A y\right) \\
= & {[A, B]+(-1)^{|x||y|}[B, A]+\frac{1}{2}\left(A y-(-1)^{|x||y|} B x\right) } \\
& +\frac{1}{2}\left((-1)^{|x||y|} B x-A y\right) \\
= & 0 .
\end{aligned}
$$

Conditions $(b),(c),(e)$ and $(f)$ are easy to be checked. By Proposition 3.2, we have $l_{3}=J_{2}$, thus Conditions $(g)-(h)$ hold. For Condition $(i)$, we first verify a special case by taking $e_{1}=A, e_{2}=B, e_{3}=C, e_{4}=w$. In fact, by the definition 
of $l_{3}$ and Proposition 3.2, we get

$$
\begin{aligned}
& {\left[A, l_{3}(B, C, w)\right]-(-1)^{|x||y|}\left[B, l_{3}(A, C, w)\right] } \\
& +(-1)^{(|x|+|y|)|z|}\left[C, l_{3}(A, B, w)\right]-\left[l_{3}(A, B, C), w\right] \\
& -l_{3}([A, B], C, w)+(-1)^{|y||z|} l_{3}([A, C], B, w)-(-1)^{(|y|+|z|)|w|} l_{3}([A, w], B, C) \\
& -l_{3}(A,[B, C], w)+(-1)^{|z||w|} l_{3}(A,[B, w], C)-l_{3}(A, B,[C, w]) \\
= & -\frac{1}{8} A[B, C] w+\frac{1}{8}(-1)^{|x||y|} B[A, C] w-\frac{1}{8}(-1)^{(|x|+|y|)|z|} C[A, B] w+0 \\
& +\frac{1}{4}[[A, B], C] w-\frac{1}{4}(-1)^{|y||z|}[[A, C], B] w+\frac{1}{4}(-1)^{|x||y|}(-1)^{|x||z|}[[B, C], A] w \\
& +\frac{1}{8}(-1)^{|x|(|y|+|z|)}[B, C] A w-\frac{1}{8}(-1)^{|y||z|}[A, C] B w+\frac{1}{8}[A, B] C w \\
= & \frac{1}{4}\left\{[[A, B], C]+(-1)^{|x|(|y|+|z|)}[[B, C], A]+(-1)^{(|x|+|y|)|z|}[[C, A], B]\right\} w \\
& -\frac{1}{8}\left\{A[B, C]-(-1)^{|x||y|} B[A, C]+(-1)^{(|x|+|y|)|z|} C[A, B]\right. \\
& \left.-(-1)^{|x|(|y|+|z|)}[B, C] A+(-1)^{|y||z|}[A, C] B-[A, B] C\right\} w \\
= & \frac{1}{4}\left\{[[A, B], C]-[A,[B, C]]+(-1)^{|x||y|}[B,[A, C]]\right\} w \\
& -\frac{1}{8}\left\{[A,[B, C]]-(-1)^{|x||y|}[B,[A, C]]-[[A, B], C]\right\} w \\
= & 0 .
\end{aligned}
$$

The general case can be checked similarly.

A Lie 2-superalgebra is called strict if $l_{3}=0$. This kind of Lie 2-superalgebras can be described in terms of crossed module.

Definition 4.3. A crossed module of Lie superalgebras consists of a pair of Lie superalgebras $\left(\mathfrak{g},[\cdot, \cdot]_{\mathfrak{g}}\right)$ and $\left(\mathfrak{h},[\cdot, \cdot]_{\mathfrak{h}}\right)$ together with an action of $\mathfrak{g}$ on $\mathfrak{h}$ and a homomorphism $\varphi: \mathfrak{h} \rightarrow \mathfrak{g}$ such that

$$
\varphi(x h)=[x, \varphi(h)]_{\mathfrak{g}}, \quad \varphi(h) k=[h, k]_{\mathfrak{h}}, \quad \forall h, k \in \mathfrak{h}, \forall x \in \mathfrak{g} .
$$

Proposition 4.4. Strict Lie 2-superalgebras are in one-to-one correspondence with crossed modules of Lie superalgebras.

Proof. Let $\mathcal{V}^{1} \stackrel{d}{\longrightarrow} \mathcal{V}^{0}$ be a strict Lie 2-superalgebra. Define $\mathfrak{g}=\mathcal{V}^{0}, \mathfrak{h}=\mathcal{V}^{1}$, and the following two brackets on $\mathfrak{g}$ and $\mathfrak{h}$ :

$$
\begin{aligned}
& {[h, k]_{\mathfrak{h}}=l_{2}(d h, k)=[d h, k], \quad \forall h, k \in \mathfrak{h}=\mathcal{V}^{1} ;} \\
& {[x, y]_{\mathfrak{g}}=l_{2}(x, y)=[x, y], \quad \forall x, y \in \mathfrak{g}=\mathcal{V}^{0} .}
\end{aligned}
$$

Obviously, $\left(\mathfrak{g},[\cdot, \cdot]_{\mathfrak{g}}\right)$ is a Lie superalgebra by $(a)$ and $(g)$ in Definition 4.1. By Condition $(h)$, we have

$$
\begin{aligned}
& -\left[[h, k]_{\mathfrak{h}}, l\right]_{\mathfrak{h}}+(-1)^{|k||l|}\left[[h, l]_{\mathfrak{h}} k\right]_{\mathfrak{h}}+\left[h,[k, l]_{\mathfrak{h}}\right]_{\mathfrak{h}} \\
= & -[d[d h, k], l]+(-1)^{|k||l|}[d[d h, l], k]+[d h,[d k, l]] \\
= & -[[d h, d k], l]+(-1)^{|k||l|}[[d h, d l], k]+[d h,[d k, l]] \\
= & 0 .
\end{aligned}
$$


This means that $\left(\mathfrak{h},[\cdot, \cdot]_{\mathfrak{h}}\right)$ is also Lie superalgebra. By Condition (e) and taking $\varphi=d$, we have

$$
\varphi\left([h, k]_{\mathfrak{h}}\right)=d([d h, k])=[d h, d k]=[\varphi(h), \varphi(k)]_{\mathfrak{g}},
$$

which implies that $\varphi$ is a homomorphism of Lie superalgebras. Next we define an action of $\mathfrak{g}$ on $\mathfrak{h}$ by

$$
x h \triangleq l_{2}(x, h)=[x, h] \in \mathfrak{h},
$$

which is an action because the equality,

$$
\begin{aligned}
& {[x, y] h-x(y h)+(-1)^{|x||y|} y(x h) } \\
= & {[[x, y], h]-[x,[y, h]]+(-1)^{|x||y|}[y,[x, h]] } \\
= & 0,
\end{aligned}
$$

holds by Condition $(h)$. Finally, it is easy to check that

$$
\begin{aligned}
\varphi(x h) & =d([x, h])=[x, d h]=[x, \varphi(h)]_{\mathfrak{g}} \\
\varphi(h) k & =[d h, k]=[h, k]_{\mathfrak{h}} .
\end{aligned}
$$

Therefore, we obtain a crossed module of Lie superalgebras.

Conversely, a crossed module of Lie superalgebras gives rise to a Lie 2superalgebra with $d=\varphi, \mathcal{V}^{0}=\mathfrak{g}, \mathcal{V}^{1}=\mathfrak{h}, l_{3}=0$ and the following operations:

$$
\begin{aligned}
& l_{2}(x, y) \triangleq[x, y]_{\mathfrak{g}}, \quad \forall x, y \in \mathfrak{g} ; \\
& l_{2}(x, h) \triangleq x h, \quad \forall x \in \mathfrak{h} ; \\
& l_{2}(h, k) \triangleq 0 .
\end{aligned}
$$

All of the conditions for a Lie 2-superalgebra can be verified directly from the definition of a crossed module.

Another kind of Lie 2-superalgebras is called skeletal if $d=0$. As pointed in [8], Skeletal Lie 2-superalgebras are in one-to-one correspondence with quadruples $\left(\mathfrak{g}, V, \rho, l_{3}\right)$ where $\mathfrak{g}$ is a Lie superalgebra, $V$ is a super vector space, $\rho$ is a representation of $\mathfrak{g}$ on $V$ and $l_{3}$ is a 3-cocycle on $\mathfrak{g}$ with values in $V$. See [14] for more details of the cohomology of Lie superalgebras.

Example 4.5. Given a quadratic Lie superalgebra $(\mathfrak{g},[\cdot, \cdot], B)$ over $\mathbb{K}$, where $B$ is the supertrace $B(x, y)=\operatorname{str}(x y)$ by Kac [7], a skeletal Lie 2-superalgebra can be constructed as follows: $\mathcal{V}^{1}=\mathbb{K}, \mathcal{V}^{0}=\mathfrak{g}, d=0$ and

$$
l_{2}(x, y)=[x, y], \quad l_{2}(x, h)=0, \quad l_{3}(x, y, z)=B([x, y], z)
$$

where $x, y, z \in \mathfrak{g}, h \in \mathbb{K}$. Condition $(i)$ is from the fact that Cartan 3 -form $l_{3}$ is closed. That is, by the invariance of $B$, we have

$$
\begin{aligned}
& \delta l_{3}(w, x, y, z) \\
= & B(w,[x,[y, z]])+B(w,[x,[y, z]])+(-1)^{|y||z|} B(w,[[x, z], y]) \\
& +(-1)^{(|x|+|y|)|z|} B(w,[z,[x, y]])-B(w,[[x, y], z])-(-1)^{|x||y|} B(w,[y,[x, z]]) \\
= & 2 B\left(w,[x,[y, z]]+(-1)^{|y||z|}[[x, z], y]-[[x, y], z]\right) \\
= & 0
\end{aligned}
$$


which holds from the super Jacobi identity on $\mathfrak{g}$. Therefore, $\left(\mathbb{K} \stackrel{0}{\longrightarrow} \mathfrak{g}, l_{2}, l_{3}\right)$ is a Lie 2-superalgebra as a super version of the string Lie algebra.

\section{References}

[1] Albeverio S, Ayupov S A, Omirov B A. On nilpotent and simple Leibniz algebras. Comm in Algebra, 2005, 33: 159-172

[2] Baez J, Crans A S. Higher-dimensional algebra VI: Lie 2-Algebras. Theo Appl Cat, 2004, 12:492-528

[3] Barreiro E, Benayadi S. Quadratic symplectic Lie superalgebras and Lie bi-superalgebras. J Algebra, 2009, 321: 582-608

[4] Bursztyn H, Cavalcanti G, Gualteri M. Reduction of Courant algebroids and generalized complex structures. Adv Math, 2007, 211: 726-765

[5] Chen Z, Liu Z J. Omni-Lie algebroids. J Geom Phys, 2010, 60:799-808

[6] Chen Z, Liu Z J, Sheng Y. Dirac structures of omni-Lie algebroids. Int J Math, 2011, 22: 1163-1185

[7] Kac V G. Lie superalgebras. Adv Math, 1977, 26: 8-96

[8] Huerta J. Division Algebras, Supersymmetry and Higher Gauge Theory. Ph.D. thesis, University of California, 2011. arXiv:1106.3385

[9] Kinyon K, Weinstein A. Leibniz algebras, Courant algebroids, and multiplications on reductive homogeneous spaces. Amer. J. math., 2001, 123: $525-550$

[10] Lada T, Stasheff J. Introduction to sh Lie algebras for physicists. Int J Theor Phys, 1993, 32: 1087-1103

[11] Liu Z J. Some remarks on Dirac structures and Poisson reductions. In Poisson geometry (Warsaw, 1998), volume 51 of Banach Center Publ., pages 165-173. Polish Acad. Sci., Warsaw, 2000.

[12] Loday J L. Une version non commutative des algèbres de Lie: les algèbres de Leibniz. Enseign Math, 1993, 39: 269-293

[13] Roytenberg D, Weinstein A. Courant Algebroids and Strongly Homotopy Lie Algebras. Lett Math Phys, 1998, 46: 81-93

[14] Scheunert M. The Theory of Lie Superalgebras. Lecture Note in Mathematics 716, Springer, 1979.

[15] Sheng Y, Liu Z J, Zhu C C. Omni-Lie 2-algebras and their Dirac structures. J Geom Phys, 2011, 61: 560-575 
[16] Uchino K. Courant brackets on noncommutative algebras and omni-Lie algebras. Tokyo J Math, 2007, 30: 239-255

[17] Weinstein A. Omni-Lie Algebras. RIMS Kôkyûroku 2000, 1176: 95-102 